YIA1

\section{IMAGING BEYOND THE LUMEN: NEAR INFRA-RED IN VIVO MOLECULAR IDENTIFICATION OF OXIDISED LDL IN ATHEROSCLEROSIS USING MAB LO1, AND THE GENERATION AND DEVELOPMENT OF ITS MOLECULARLY EXPRESSED CYSTEINE-TAGGED CHIMERIC FAB CONSTRUCT (LO1-FAB-CYS)}

R Khamis, ${ }^{1}$ K Woollard, ${ }^{1}$ D Granger, ${ }^{2}$ Shang-Hung Chang, ${ }^{1} \mathrm{~J}$ J Boyle, ${ }^{1}$ V M Johns, P M Matthews, ${ }^{1}$ D 0 Haskard ${ }^{1}{ }^{1}$ mperial College London; ${ }^{2}$ Biopharm RED, GlaxoSmithKline

\section{doi:10.1136/heartjin-2013-304019.268}

Introduction The in vivo identification of vulnerable atherosclerotic plaque remains elusive despite current advances in intravascular morphological imaging technologies such as IVUS-VH and OCT. With the recent advent of intra-vascular catheter-based optical fluorescence domain imaging combined with near infra-red fluorescence (OFDI-NIRF), we aim to translationally develop a novel NIRF antibody-based technique for the identification of oxidised LDL in atherosclerosis.

Methods and Results We isolated a previously unknown IgG3к autoantibody against oxLDL (designated LO1) from an LDL receptor deficient hypercholesterolaemic mouse. Characterisation of LO1 revealed specificity for malondialdehyde-conjugated LDL (MDA-LDL), an epitope expressed in oxidised LDL and which is known to reflect vulnerability in human atherosclerotic lesions. Immunohistochemical (IHC) staining of biotinylated LO1 showed binding to advanced mouse atherosclerotic plaque and to necrotic core within culprit human carotid atherosclerotic plaque. We used the immunoglobulin $\mathrm{SH}$ groups to successfully label LO1 with a Near Infra-Red Fluorescence (NIRF) reporter VivoTag-S MAL 750 (designated LO1-750) for in vivo imaging studies. Using two optical imaging techniques (IVIS Spectrum combined with hybrid high resolution CT; Fluorescence Molecular Tomography (FMT)) we have demonstrated that intra-venously injected LO1-750 sensitively and specifically identifies atherosclerotic lesions in vivo in the aortae of $\mathrm{LDLr}^{-/-}$ mouse model (Figure 1) but not in control mice. LO1-750 also demonstrated superior specificity for lesional regions of interest (ROIs) than a caged MMP probe. In situ en face IHC on extracted aortae with LO1-750 showed localisation to the aortic arch and root, with co localisation to the sub-endothelium as demonstrated by counter injection with an anti CD-31 antibody (Figure 2).

In order to facilitate future use in human subjects, LO1 was successfully sequenced and sequence verified as germline. It was then

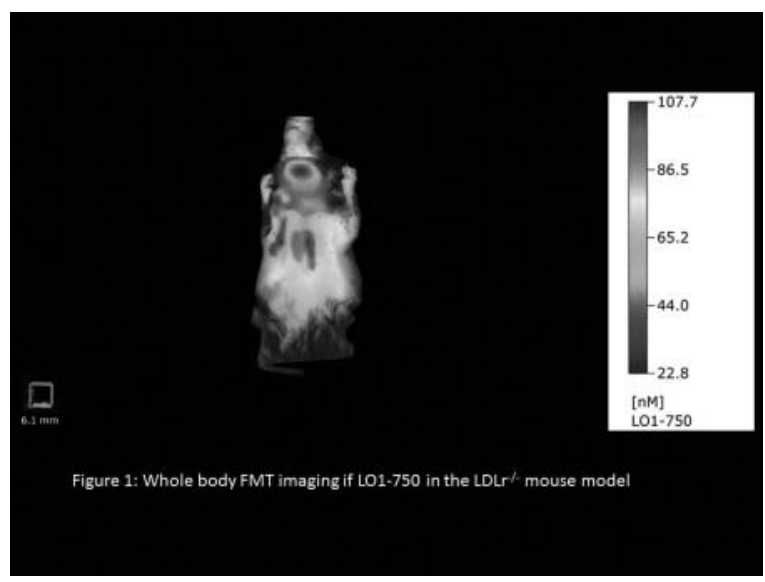

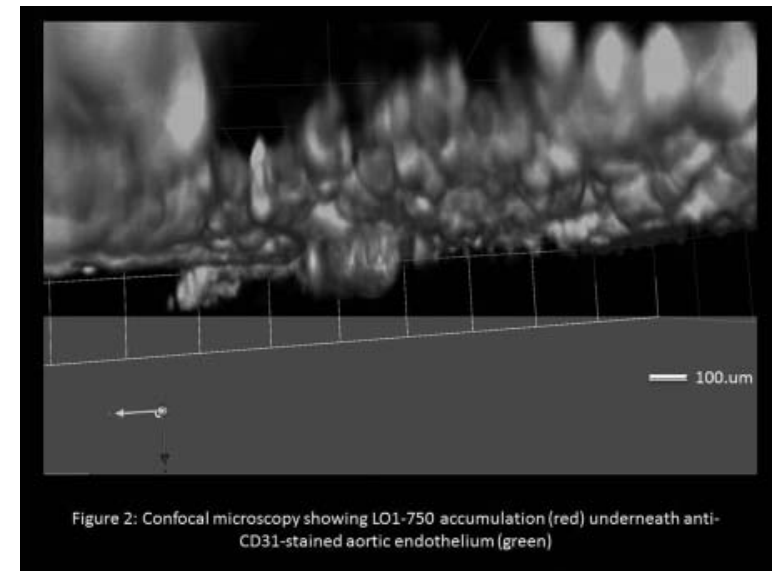

Figure 2

expressed in HEK cells as a chimeric Fab $(\sim 50 \mathrm{kD})$, with murine $\mathrm{VH}$ and $\mathrm{VL}$ and human $\mathrm{CH} 1$ and $\mathrm{CL}$. The terminus of $\mathrm{CH} 1$ extends to the THTC of the hinge region to provide the free cysteine for NIRF labelling. In vitro characterisation of the construct revealed maintained function when purified over an anti-human $\mathrm{CH} 1$ column and labelled with Vivo Tag-S MAL 750. Injected LO1-cys-fab localised to the sub-endothelium of the diseased aortic arch of $\mathrm{LDLr}^{-/-}$mice in a similar fashion to the native $\mathrm{LO} 1$ antibody. Initial FMT studies have confirmed localisation to atherosclerotic lesions within the $\mathrm{LDLr}^{-/-}$model.

Conclusion The in vivo optical imaging of oxidised $\mathrm{LDL}$ is possible with LO1 and the chimerically humanised LO1-Cys-Fab. Future work will aim to translate this to optical imaging of atherosclerosis in humans. 